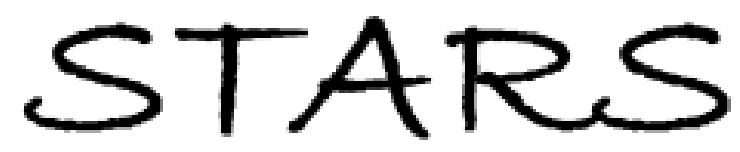

University of Central Florida

STARS

Faculty Bibliography 2000s

Faculty Bibliography

$1-1-2009$

\title{
Diffractive optical elements for pitchfork beam shaping
}

Chong Zhang

University of Central Florida

Nathaniel R. Quick

Aravinda Kar

University of Central Florida

Find similar works at: https://stars.library.ucf.edu/facultybib2000

University of Central Florida Libraries http://library.ucf.edu

This Article is brought to you for free and open access by the Faculty Bibliography at STARS. It has been accepted for inclusion in Faculty Bibliography 2000s by an authorized administrator of STARS. For more information, please contactSTARS@ucf.edu.

\section{Recommended Citation}

Zhang, Chong; Quick, Nathaniel R.; and Kar, Aravinda, "Diffractive optical elements for pitchfork beam shaping" (2009). Faculty Bibliography 2000s. 2377.

https://stars.library.ucf.edu/facultybib2000/2377

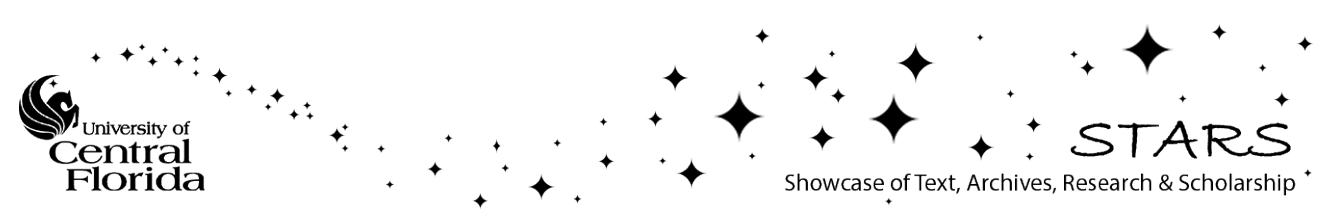




\section{Diffractive optical elements for pitchfork beam shaping}

\author{
Chong Zhang \\ University of Central Florida \\ CREOL, The College of Optics and Photonics \\ Department of Mechanical, Materials and \\ Aerospace Engineering \\ Laser Advanced Materials Processing Laboratory \\ P.O. Box 162700 \\ 4000 Central Florida Boulevard \\ Orlando, Florida 32816-2700
}

Nathaniel R. Quick

Applicote Associates, LLC

1445 Dolgner Place, Suite 23

Sanford, Florida 32771

\author{
Aravinda Kar \\ University of Central Florida \\ CREOL, The College of Optics and Photonics \\ Department of Mechanical, Materials and \\ Aerospace Engineering \\ Laser Advanced Materials Processing Laboratory \\ P.O. Box 162700 \\ 4000 Central Florida Boulevard \\ Orlando, Florida 32816-2700 \\ E-mail: akar@creol.ucf.edu
}

\begin{abstract}
A set of laser beam shaping optics is designed by an iterative method using an adaptive additive algorithm to transform a Gaussian beam into a pitchfork beam. Two diffractive optical elements are designed based on Fresnel diffraction to reduce the amount of energy in the first-order diffraction ring and to increase the depth of focus for the optical system. These two beam properties are found to depend on the diameter of the desired beam and the Airy disk diameter. If the diameter of the desired beam is large, then the optical system yields better results in achieving the above-mentioned two beam properties. The performance of the diffractive optical elements is compared to a previous laser beam shaping system designed by the ray-tracing technique. A pinhole scanning power meter is used to measure the laser irradiance profile at the focal plane to verify the existence of the pitchfork beam. The irradiance profile measurement shows that diffractive optical elements allow better control for reducing the amount of energy in the diffraction side lobes. () 2009 Society of Photo-Optical Instrumentation Engineers. [DOI: 10.1117/1.3183919]
\end{abstract}

Subject terms: laser beam shaping; diffractive optics; lens design; pitchfork beam; laser microvia drilling.

Paper 090181R received Mar. 16, 2009; revised manuscript received May 21 2009; accepted for publication May 30, 2009; published online Jul. 24, 2009.

\section{Introduction}

The Gaussian mode of a laser beam is the lowest order transverse electromagnetic, (i.e., $\mathrm{TEM}_{00}$ ) mode of an optical oscillator producing the beam. The beam quality factor $\left(M^{2}\right)$ is 1 for a perfect Gaussian mode, indicating that the laser beam has the highest possible beam quality. The irradiance of Gaussian laser beams is given by the expression $I(r)=I_{0} \exp \left[-2\left(r / r_{0}\right)^{2}\right]$, where $I(r)$ is the laser irradiance at any radial point $r$ from the center of the laser beam on a transverse plane, $I_{0}$ is the irradiance at the center of the laser beam, and $r_{0}$ is the laser beam radius at $1 / e^{2}$-point. The laser irradiance is maximum at the center of Gaussian beams and decreases toward the edge of the beams. Different irradiance profiles are, however, necessary in many applications. Laser beam shaping is the process of converting the irradiance and phase of the laser into the desired profile. ${ }^{1}$ It is widely used in laser materials processing, such as pulsed laser deposition, lithography, micromachining, ${ }^{2}$ laser fusion, laser radar, laser scanning, and optical data processing. ${ }^{3}$ For different laser materials processing applications, different irradiance profiles, such as flat-top beam, Bessel beam, and annular beam ${ }^{4-6}$ have been utilized. The easiest approach in shaping a beam is the use of apodization and truncation. However, this approach is fundamen-

0091-3286/2009/\$25.00 @ 2009 SPIE tally energy inefficient. Reflective optics, refractive optics, diffractive optics, ${ }^{7}$ acousto-optics, ${ }^{8}$ and liquid crystal $^{9}$ are generally employed to shape laser beams.

Flat-top beams are currently used in laser microvia drilling application. Zhang et al. ${ }^{10}$ designed a set of optics based on ray tracing to transform a Gaussian beam into a pitchfork beam to improve the microvia quality, i.e., smaller via diameter, less taper, and less corner residue than what are obtained by flat-top beam drilling. The ray-tracing technique utilizes refraction in geometric optics to redistribute the laser irradiance without considering the wave property of the lasers. As a result, although the ray-tracing approach indicated no diffraction ring to be produced by their ${ }^{10}$ optics, the experimental data proved otherwise with a diffraction ring of radius $30 \mu \mathrm{m}$. Diffractive analysis is necessary to redistribute the laser energy with better precision. When the laser wavelength is large or the diameter of the focused laser beam is small, the diffraction effect becomes important for drilling microvias of small diameters. $\mathrm{CO}_{2}$ lasers of wavelength $9.3 \mu \mathrm{m}$ are usually employed in laser microvia drilling industries because such lasers allow drilling at high throughput rates. Because of the relatively long $(9.3-\mu \mathrm{m})$ wavelength and small size of the microvias $(\sim 60 \mu \mathrm{m})$, the Fresnel diffraction analysis is considered for designing beam-shaping optics in this paper. The function of this new set of optics is to reduce the amount of energy in the first diffraction ring and to increase the depth of focus. Various algorithms, such as iterative Fourier 


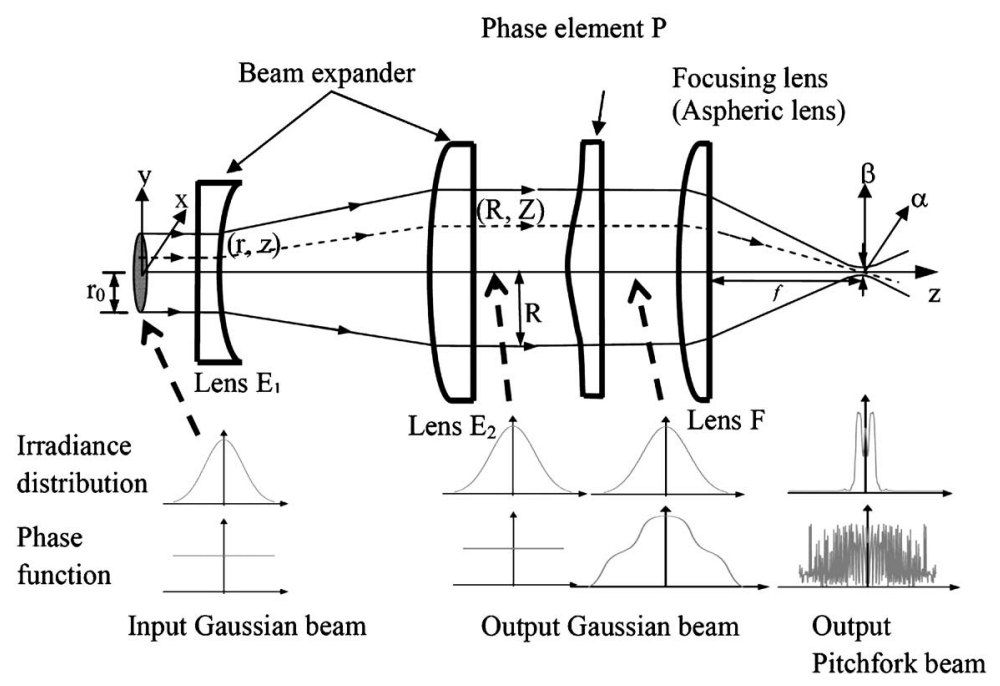

Fig. 1 Optical system for pitchfork beam shaping.

transform, ${ }^{11}$ Gerchberg-Saxton (GS) ${ }^{12,13}$ method, direct binary search, ${ }^{14}$ and simulated annealing, ${ }^{15,16}$ are often used for laser beam shaping in free space. Using a repeated Fourier transform algorithm for the near- and far-field planes, Dixit et al. ${ }^{17,18}$ obtained a super-Gaussian beam with more than $95 \%$ of the incident energy contained in the central portion of the beam. Lin et al. ${ }^{19}$ employed the GS algorithm and obtained a super-Gaussian beam with low (5-10\%) scattering loss. Liu and Taghizadeh ${ }^{20}$ developed an improved iterative algorithm based on the GS approach and obtained higher-order super-Gaussian beams with $97.4 \%$ of the incident energy contained in the central portion of the beam. Guang et al. ${ }^{21}$ used the stationary phase method to design the diffractive optical flat-top beam shaper. The beam shaper was fabricated by creating a dark silver ion exchange layer on a high-energy beam-sensitive glass with an electron beam and then etching the layer using a laserassisted chemical etching method. They experimentally observed that the energy conversion efficiency of their beam shaper can reach $82 \%$. In the present work, an adaptive additive algorithm is used in an iterative scheme for designing diffractive optical elements to produce a central lobe containing more laser energy than in the case of usual central lobes produced by conventional optics. The designed diffractive optical elements were fabricated using the diamond turning method to investigate whether this method can be applied to produce beam-shaping optics, which, incidentally, reduces the cost of fabricating such diffractive optical elements.

\section{Transformation of a Gaussian Beam into a Pitchfork Beam}

Figure 1 shows the optical elements investigated in this study for an incident laser beam having a planar phase function. A beam expander (lenses $E_{1}$ and $E_{2}$ ) of expansion ratio 10 is placed before the phase element $\mathrm{P}$ to create a laser spot of small Airy disk diameter $(23 \mu \mathrm{m})$ on the focal plane of the focusing lens F (Fig. 1). The beam expander expands the laser beam while still maintaining the planar phase function of the incident beam. The phase element introduces a phase shift to the expanded Gaussian beam and thus alters the shape of the wavefront. This phase shift is so designed using the diffraction theory that the expanded Gaussian beam can be focused with a $50.8 \mathrm{~mm}$ focal-length lens into a pitchfork beam on the focal plane. The desired pitchfork amplitude profile, $U_{p}(r)$, is shown in Fig. 2 and the pitchfork beam diameter is considered approximately twice the Airy disk diameter.

The phase element $\mathrm{P}$ is optimized by an iterative method using adaptive additive algorithm. ${ }^{22}$ Generally, diffractive optical elements are designed based on Fourier optics by introducing different spatial phase functions so that the optical waves of identical beam profile (such as the Gaussian profile) can produce different profiles on the image plane. In beam-shaping applications, ${ }^{10}$ the irradiance profile of the laser source and the desired irradiance profile on the image plane are known, and the unknown is the phase function. An adaptive additive algorithm provides a convenient iterative approach involving the Fourier transform to determine

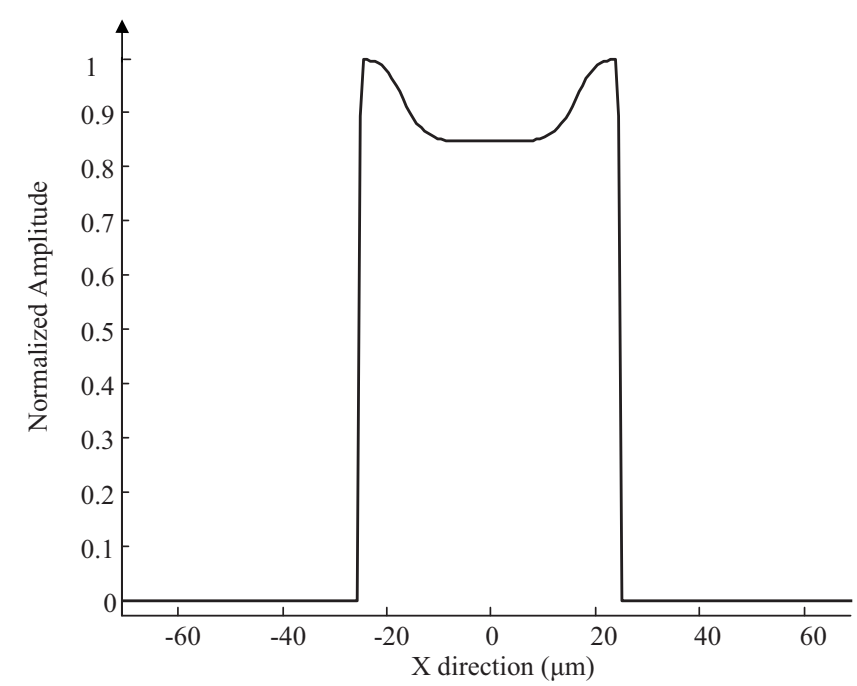

Fig. 2 Desired amplitude of the pitchfork beam 


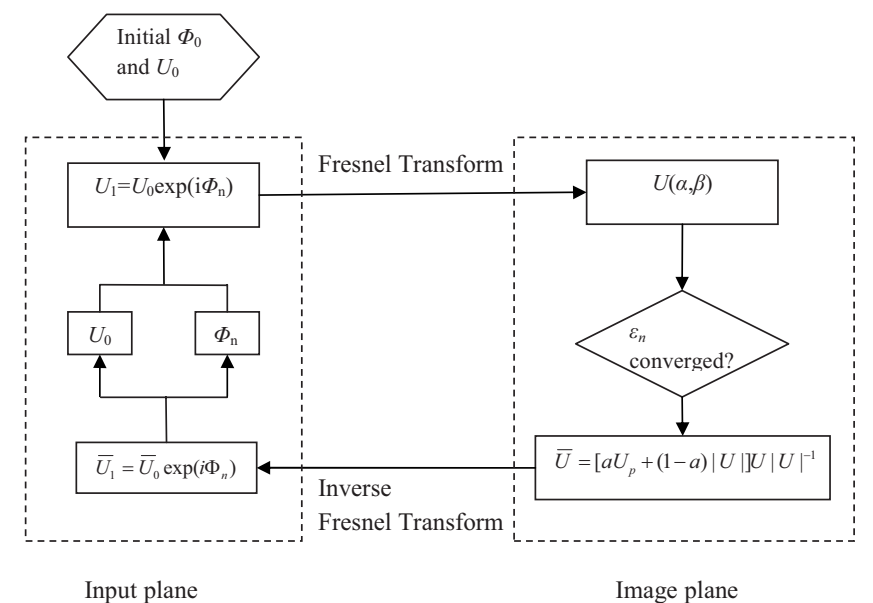

Fig. 3 Flowchart for the adaptive-additive algorithm

the phase function for beam shaping. Basically, this algorithm enables solving the following integral equation by the method of successive approximation

$$
\begin{aligned}
U(\alpha, \beta, L)= & \iint_{-\infty}^{\infty} U_{0}(x, y) \exp [i \Phi(x, y)] \\
& \times H(x-\alpha, y-\beta, L) d x d y,
\end{aligned}
$$

where $U(\alpha, \beta, L)$ is the desired amplitude distribution that should be produced by the phase element $\mathrm{P}$ and the Fourier transform element (focusing lens F in Fig. 1) at a distance $L$. If $L$ is constant, then the amplitude can be written as $U(\alpha, \beta) . L$ is measured in the longitudinal direction (i.e., in the direction of the wave propagation), and $(\alpha, \beta)$ represents an arbitrary coordinate point in the transverse plane $\alpha-\beta$ in the Cartesian coordinates as shown in Fig. 1 . $U_{0}(x, y)$ is the amplitude of the incident light at the phase element $\mathrm{P}$ in the transverse plane $x-y$, and $\Phi(x, y)$ is the desired phase function that needs to be introduced into the incident light by the phase element. $H(x-\alpha, y-\beta, L)$ is the Green's function, i.e., the impulse response representing the wave at a point $(\alpha, \beta, L)$ due to an impulse source at $(x, y, 0)$ denoted by the Dirac $\delta$ function $\delta(x, y, 0)$, which is also known as the propagation function in free space. ${ }^{22}$ In the Fresnel diffraction approximation, it has the following expression:

$H(x, y, L)=\frac{k}{z} \exp \left[\frac{i k}{2 z}\left(x^{2}+y^{2}\right)\right]$,

where $k=2 \pi / \lambda$ is the spatial frequency and $\lambda$ is the wavelength of light in free space.

The adaptive additive algorithm, which is illustrated in Fig. 3, starts with an initial value $\Phi_{0}(x, y)$ and an initial input wavefront, $U_{0}(x, y)$, which is a Gaussian profile in this study, resulting in the following wavefront:

$U_{1}(x, y)=U_{0}(x, y) \exp \left[i \Phi_{0}(x, y)\right]$.

The Fresnel transform of this wavefront is the first estimate for the output electric field on the image plane

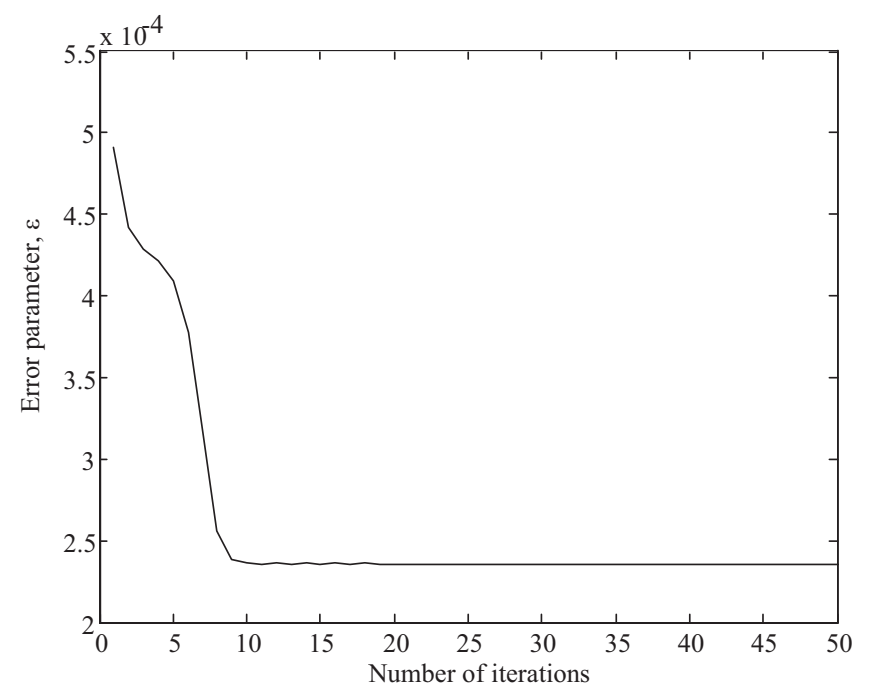

Fig. 4 Error parameter as a function of number of iterations

$U(\alpha, \beta)=\int_{-\infty}^{\infty} \int_{-\infty}^{\infty} U_{1}(x, y) H(x-\alpha, y-\beta, L) d x d y$.

Normally, this amplitude differs from the desired amplitude profile $U_{\mathrm{p}}(\alpha, \beta)$. The convergence of the iterative process is evaluated using the following error parameter

$\varepsilon=\int_{-\infty}^{\infty} \int\left[|U(\alpha, \beta)|-U_{\mathrm{p}}(\alpha, \beta)\right]^{2} d \alpha d \beta$.

For a convergent iteration scheme, the amplitude profile in the inverse Fresnel transform needs to be changed gradually toward the desired beam profile in each iteration step. This is accomplished by adding a weighted amount, $a$, of the desired amplitude to the field in the image plane

$\bar{U}(\alpha, \beta)=\left[a U_{\mathrm{p}}(\alpha, \beta)+(1-a)|U(\alpha, \beta)|\right] U(\alpha, \beta)|U(\alpha, \beta)|^{-1}$,

where the weighted amount, $a$, is chosen as 1.5 in this study. The inverse Fresnel transform of $\bar{U}(\alpha, \beta)$ yields the corresponding amplitude and phase function $\bar{U}_{1}(x, y)$ $=\bar{U}_{0}(x, y) \exp \left[i \Phi_{1}(x, y)\right]$ in the input plane. After this transform, the amplitude of the input wavefront usually differs from the actual incident laser profile. Thus, $\bar{U}_{0}(x, y)$ is replaced with the Gaussian profile $U_{0}(x, y)$ resulting in an improved estimate for the input field: $U_{1}(x, y)$ $=U_{0}(x, y) \exp \left[i \Phi_{1}(x, y)\right]$. These operations complete one iteration step in the adaptive additive algorithm. This iteration step is repeated until the error, $\varepsilon$, converges to within an acceptable tolerance in the $n$th iteration. Thus, the phase function, $\Phi(x, y)=\Phi_{n}(x, y)$, can be determined to ensure that the incident laser beam profile is transformed into the desired amplitude profile $U_{\mathrm{p}}(\alpha, \beta)$ on the image plane.

\section{Results and Discussions}

Figure 4 shows that the error parameter decreases as the iteration number increases, the iteration is convergent, and 


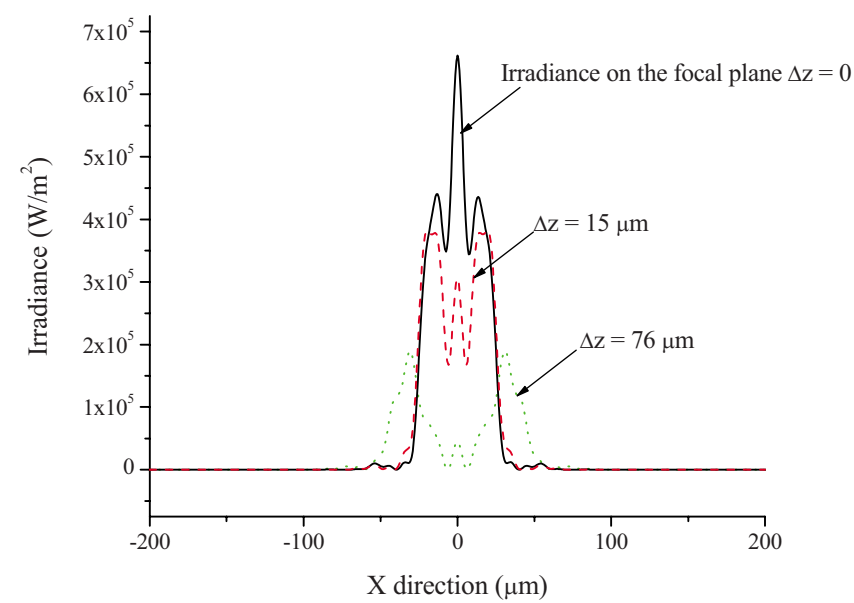

Fig. 5 Laser irradiances at different $z$ positions

the error parameter reduces to a small value very fast. After 50 iterations, the calculated irradiance distribution reached the acceptable pitchfork profile, as shown in Fig. 5. The diffraction side lobe contains only $4.7 \%$ of the incident laser energy. In laser microvia drilling applications, the peak in the periphery of the pitchfork beam can be used to remove the residue from the corner at the bottom of the vias. ${ }^{10}$ The irradiances at different $z$ positions are included in Fig. 5. The irradiance maintains a good pitchfork profile within the depth of $\pm 15 \mu \mathrm{m}$ around the focal plane. An annular irradiance profile is obtained when the $z$ position is $\pm 76 \mu \mathrm{m}$ outside the focal plane of lens $\mathrm{F}$.

\subsection{Design of the Phase Element Surface}

When the acceptable beam profile is obtained in the $\alpha-\beta$ plane (Fig. 1) by the above-mentioned iterative calculation, the design of the phase element is considered to be complete. In this study, the phase element $\mathrm{P}$ is designed to obtain a pitchfork profile at the $\alpha$ - $\beta$ plane from a Gaussian irradiance profile. The expression $\phi(x, y)=k n t(x, y)$ is used to determine the shape of the phase element, where $k$ is the wave vector, and $n$ and $t(x, y)$ are the refractive index and the thickness of the phase element respectively. A planoconvex phase element can be designed so that its curved surface represents the calculated phase function. Curve A in Fig. 6 represents the calculated shape of the phase element, showing two discontinuities $\mathrm{A}_{2} \mathrm{~A}_{3}$ and $\mathrm{A}_{5} \mathrm{~A}_{6}$ at the positions where the values of the phase functions are 0 and $+\pi$, respectively. Such discontinuous profiles represent diffractive features, which are fabricated by chemical etching, in traditional diffractive optical elements. Because the discontinuous diffractive features are difficult to fabricate on $\mathrm{ZnSe}$, a phase shift of $\pi$ is applied to the central region $\left(\mathrm{A}_{3} \mathrm{~A}_{4} \mathrm{~A}_{5}\right)$ of curve $\mathrm{A}$ to attain a smooth surface represented by curve $B$. Shifting the phase of region $\mathrm{A}_{3} \mathrm{~A}_{4} \mathrm{~A}_{5}$ by $\pi$ does not affect the irradiance of the pitchfork profile because the irradiance is the magnitude of the phasor (i.e., the complex amplitude of the electromagnetic field of the laser beam). The continuous phase function is represented by the continuous variation in the height of the phase element (curve $\mathrm{B}$ in Fig. 6) so that it can be produced by the diamond

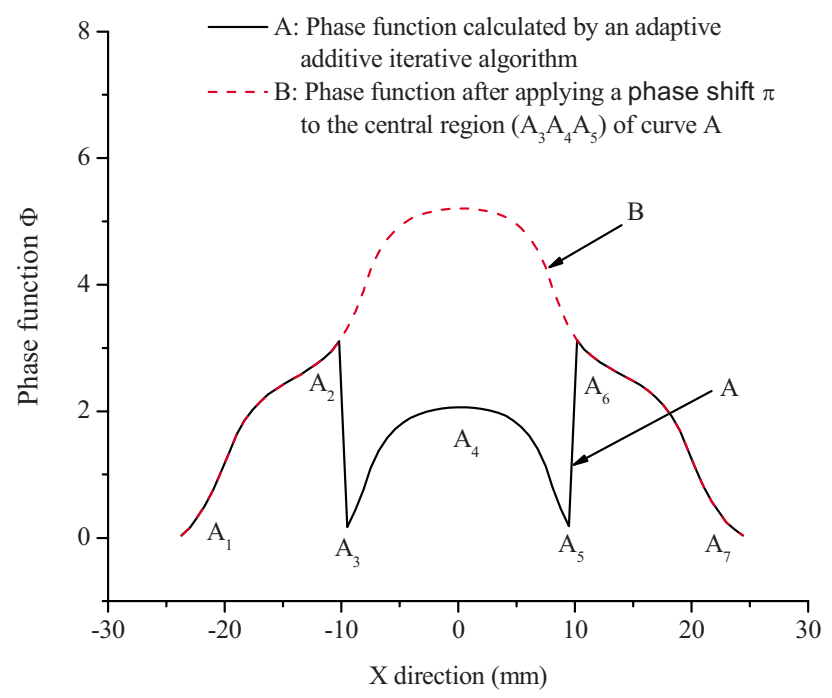

Fig. 6 Phase function introduced by the phase element

turning method. A six-order polynomial is used to fit the curvature, yielding the following equation for the surface of the phase element $\mathrm{P}$ :

$$
\begin{aligned}
Z= & -1.27 \times 10^{-1} \times R+1.18 \times 10^{-1} \times R^{2}-3.42 \times 10^{-2} \\
& \times R^{3}+3.32 \times 10^{-3} \times R^{4}-1.36 \times 10^{-4} \\
& \times R^{5}+2 \times 10^{-6} \times R^{6}
\end{aligned}
$$

where $Z$ is the height and $R$ is the radius of the phase element, and they are in units of micrometers and millimeters, respectively.

\subsection{Effect of the Airy Disk Diameter on the Optimized Phase Element Surface}

In the above-mentioned optimization of the phase element to transform a Gaussian beam into a Pitchfork beam, the pitchfork beam diameter $\left(d_{0}\right)$ is approximately twice the Airy disk diameter, $d_{a 2}$, for a $50.8 \mathrm{~mm}$ focal length lens

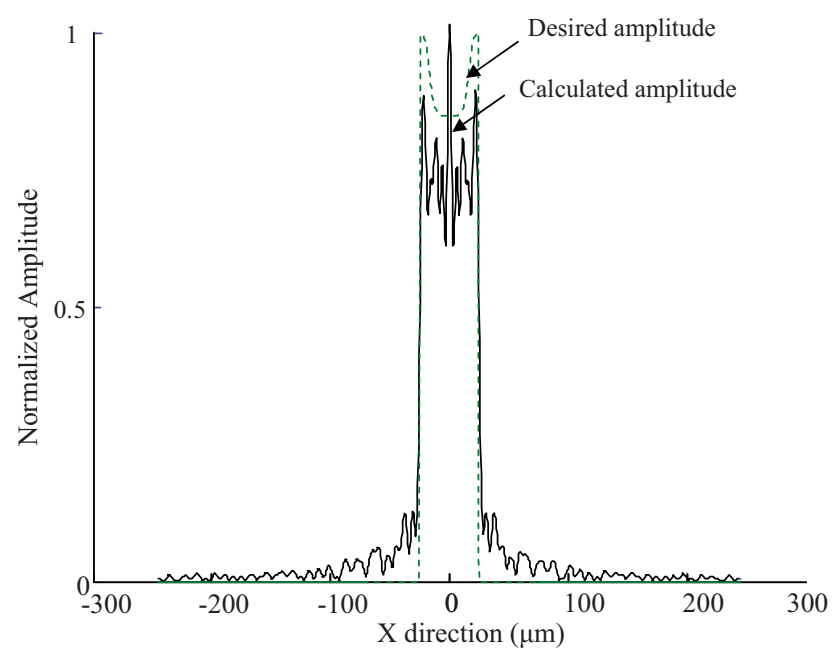

Fig. 7 Optimized amplitude of the pitchfork beam when $d_{0}=4 d_{a}$ and the focal length of lens $F$ is $25.4 \mathrm{~mm}$. 


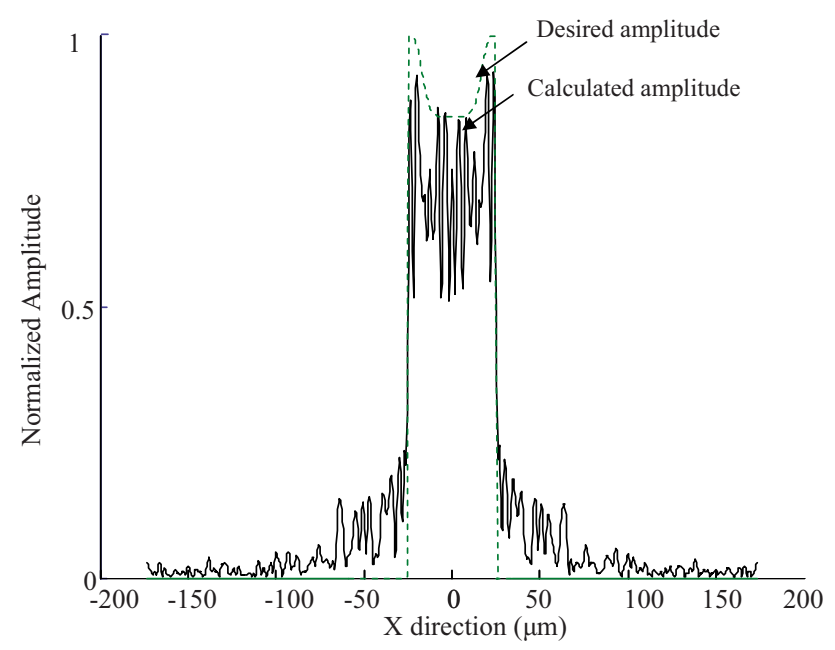

Fig. 8 Optimized amplitude of the pitchfork beam when $d_{0}=8 d_{a}$ and the focal length of lens $F$ is $12.7 \mathrm{~mm}$.

(i.e., $d_{0} \approx 2 d_{a 2}$ ). The calculated pitchfork beam resembles the desired pitchfork beam (i.e., the irradiance profile and diameter of both beams are close to each other), if the desired pitchfork beam diameter is more than twice the Airy disk diameter on the focal plane. It should be noted that the Airy disk diameter produced by a lens refers to the diameter on the focal plane due to the diffraction of a plane wave. The Airy disk diameter for this $25.4 \mathrm{~mm}$ focal length lens $\left(d_{a 1}\right)$ is one-half of the Airy disk diameter $\left(d_{a 2}\right)$ obtained above with a $50.8 \mathrm{~mm}$ focal length lens (i.e., $d_{a 1}$ $\left.=d_{a 2} / 2\right)$. The calculated and desired amplitude profiles obtained with a $25.4 \mathrm{~mm}$ focal length lens is presented in Fig. 7 for the case of the pitchfork beam diameter being approximately four times the Airy disk diameter $\left(d_{0} \approx 4 d_{a 1}\right)$. For a $12.7 \mathrm{~mm}$ focal length lens, the Airy disk diameter $d_{a 1 / 2}=d_{a 2} / 4$. As a result, when the pitchfork beam diameter is approximately eight times the Airy disk diameter $\left(d_{0}\right.$ $\approx 8 d_{a 1 / 2}$ ), Fig. 8 shows that the optimum amplitude profile matches with the desired pitchfork profile better than in Fig. 7. However, short focal-length lenses are prone to aberration and the cost of fabricating such lenses is relatively high. For these reasons, a $50.8 \mathrm{~mm}$ focal length lens is used and the pitchfork beam diameter is set twice the Airy disk diameter $\left(d_{0}=2 d_{a 2}\right)$ in this study to ease the optics fabrication process.

\subsection{Optimization for the Depth of Focus}

The depth of focus is only $30 \mu \mathrm{m}$ for the optics designed above, but a longer depth of focus such as $150 \mu \mathrm{m}$ is desirable to allow tolerance for placing the substrate at the focal plane ( $\alpha$ - $\beta$ plane in Fig. 1) in microvia production facilities. Therefore, a second phase element D (Fig. 9) is designed and inserted before the focusing lens to increase the depth of focus. This depth of focus phase element D is designed using the Fresnel diffraction theory for the purpose of producing a beam that maintains the same irradiance on the optical axis ( $z$-axis) over a certain distance $\Delta z$.

The amplitude on the optical axis ( $\rho=0$ in Fig. 9) can be calculated as

$U(0, z)=\frac{k}{z} \int_{0}^{R} U_{0}(r) \exp [i \Phi(r)] \exp \left(i \frac{k}{2 z} r^{2}\right) r d r$,

where $k$ is the wave number, $R$ is the radius of diffractive optical element, $r$ and $\rho$ are the radial variables in the diffractive optical element $(x-y)$ plane and the observation $(\alpha-\beta)$ plane. Letting $\xi=k / z$ and $x=r^{2} / 2$, Eq. (8) can be written as

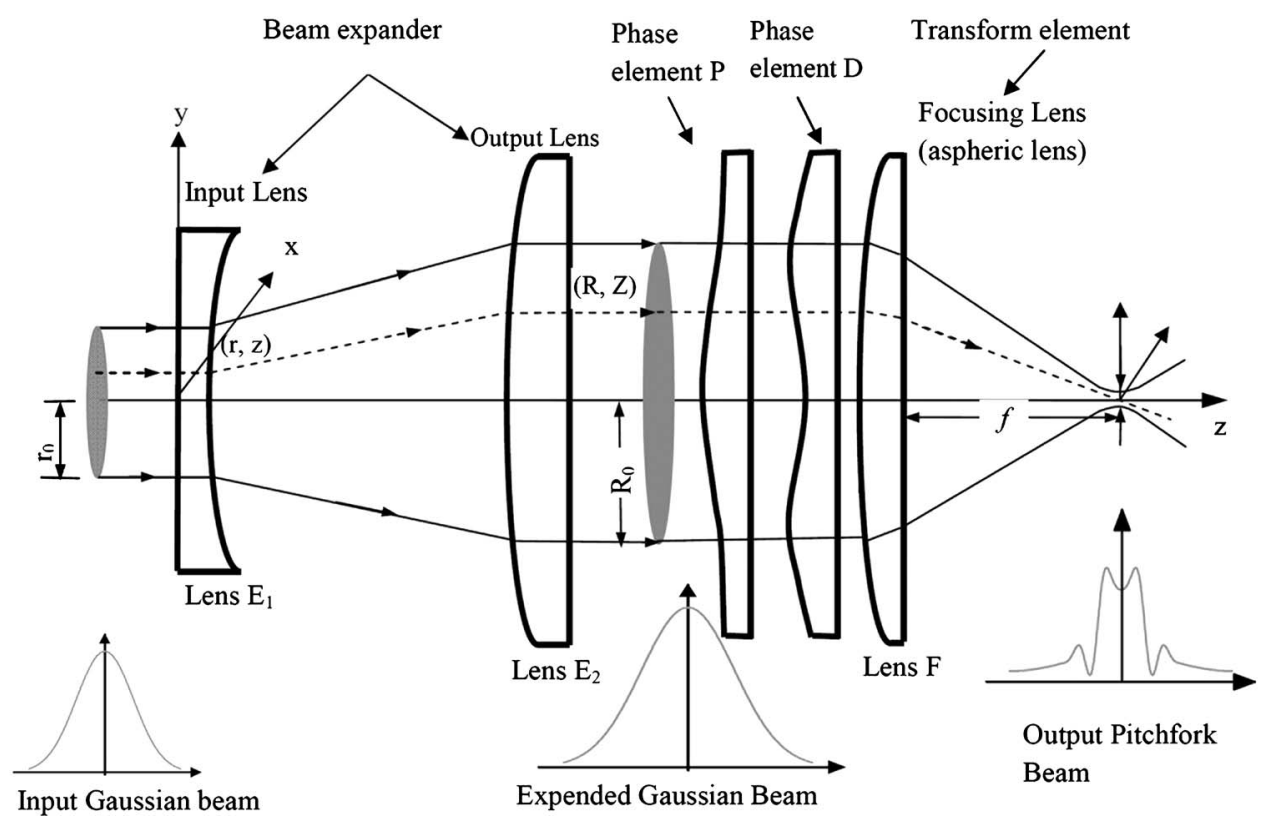

Fig. 9 Output pitchfork beam with improved depth of focus produced by the optical system. 


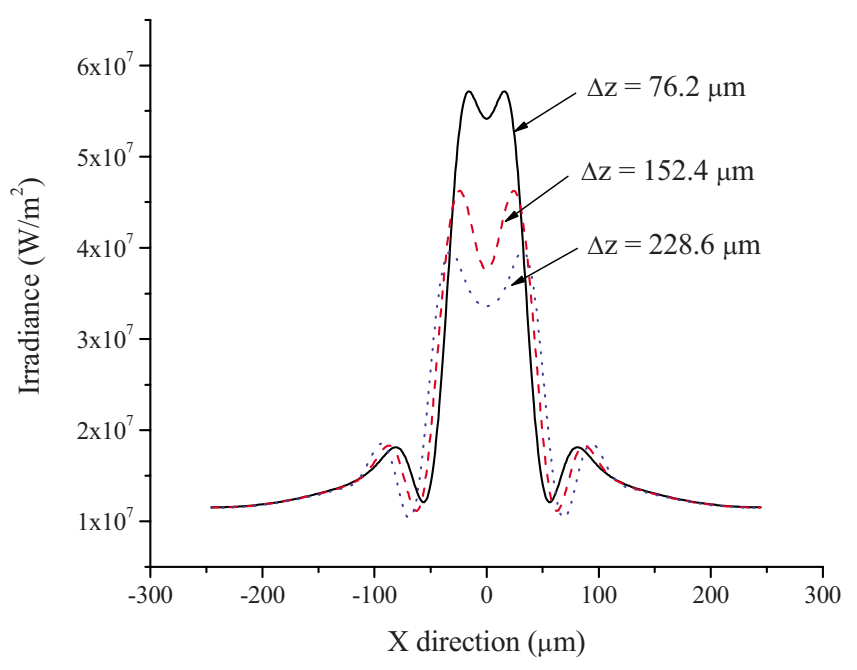

Fig. 10 Laser irradiances at different $z$ positions $(f=50.8 \mathrm{~mm})$.

$U(\xi)=\int_{0}^{x_{0}} U_{0}(x) \exp [i \Phi(x)] \exp (i x \xi) d x$.

Equation (9) is solved by the adaptive additive algorithm to obtain the phase function that produces the desired amplitude along the z-axis in Fig. 9. After optimizing the depth of focus, the irradiances are calculated for different values of $\Delta z$, (i.e., for different values of $z$ relative to the focal plane of the focusing lens F) as shown in Fig. 10. The focused beam diameter is $100 \mu \mathrm{m}$, corresponding to an optimum depth of focus $457 \mu \mathrm{m}$. Smaller beam of diameter $50 \mu \mathrm{m}$ can be achieved as shown in Fig. 11 with a focusing lens of shorter $(25.4 \mathrm{~mm})$ focal length. However, the difficulties to fabricate such short focal-length lenses need to be considered for implementation of such optics in manufacturing plants. Therefore, the phase function of the depth of focus phase element $\mathrm{D}$ was optimized for a $50.8 \mathrm{~mm}$ focal length lens and the corresponding shape of this phase element is shown in Fig. 12. A seven-order polynomial is used to represent the surface of this phase element

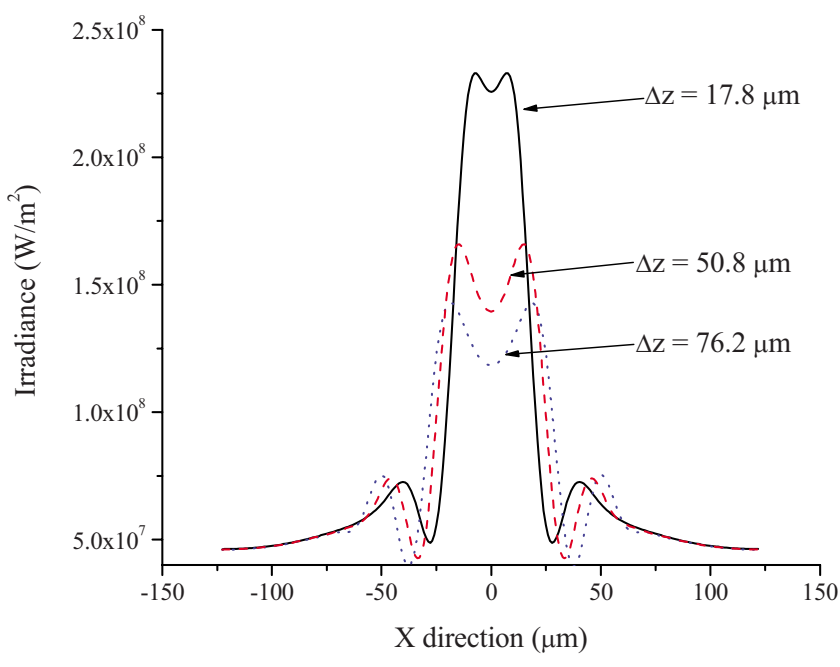

Fig. 11 Laser irradiances at different $z$ positions $(f=25.4 \mathrm{~mm})$.

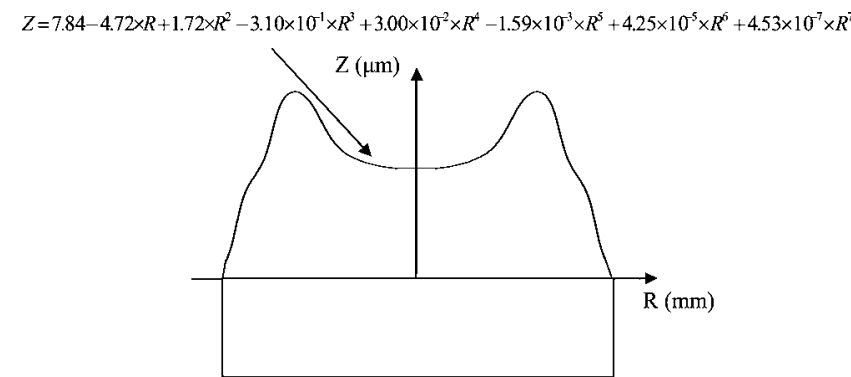

Fig. 12 Surface profile of the phase element $D$ to improve depth of focus.

$$
\begin{aligned}
Z= & 7.84-4.72 \times R+1.72 \times R^{2}-3.10 \times 10^{-1} \\
& \times R^{3}+3.00 \times 10^{-2} \times R^{4}-1.59 \times 10^{-3} \\
& \times R^{5}+4.25 \times 10^{-5} \times R^{6}+4.53 \times 10^{-7} \times R^{7},
\end{aligned}
$$

where $Z$ and $R$ are the height and radius of the phase element in units of micrometers and millimeters respectively.

The entire optical system is presented in Fig. 9, showing two phase elements $\mathrm{P}$ and $\mathrm{D}$ for beam shaping. The phase element $\mathrm{P}$ introduces a phase function to the Gaussian beam to produce an optimum pitchfork beam. The phase element $\mathrm{D}$ introduces a phase function to the beam produced by the phase element $\mathrm{P}$ in order to increase the depth of focus of the focusing lens $\mathrm{F}$.

\section{Pitchfork Beam Irradiance Profile Measurement at the Focal Plane}

To validate the performance of the phase elements in transforming a Gaussian laser beam into a pitchfork beam, the optical components in Fig. 1 were custom built by the method of diamond turning. A pinhole scanning method was developed to measure the irradiance profile of the pitchfork beam using an experimental setup shown in Fig. 13. This method is capable of measuring the profile of high irradiance beams even at the focal plane. A GEM-Q600 Q-switched $\mathrm{CO}_{2}$ laser of wavelength $9.3 \mu \mathrm{m}$ manufactured by the Coherent/DEOS company was used in this experiment. The Gaussian beam of this laser system was expanded using lenses $E_{1}$ and $E_{2}$. The phase element P introduces the desired phase function calculated by the abovementioned adaptive additive algorithm and transforms the Gaussian irradiance profile into a pitchfork irradiance profile on the focal plane of lens $\mathrm{F}$.

To build the pinhole scanning device, a 10- $\mu$ m-diam pinhole was drilled in a copper sheet of thickness $520 \mu \mathrm{m}$ with a frequency-doubled Nd:YAG laser and the sheet was placed on top of a highly sensitive power sensor capable of measuring power as low as $10 \mu \mathrm{W}$. The pinhole and sensor assembly was placed on an $X Y$ stage to achieve the scanning capability. This scanning device was placed at the plane where the pitchfork beam is formed. On the basis of the power $P$ measured by the power sensor and the area of the pinhole $A_{p}$, the irradiance profile is calculated as $I$ $=P / A_{p}$, which represents the average irradiance of a small segment of the beam that passes through the pinhole. The irradiance profile can also be measured at different $z$ positions using an $X Y Z$ stage instead of an $X Y$ stage. The designed laser beam diameter is $50 \mu \mathrm{m}$ on the focal plane of 


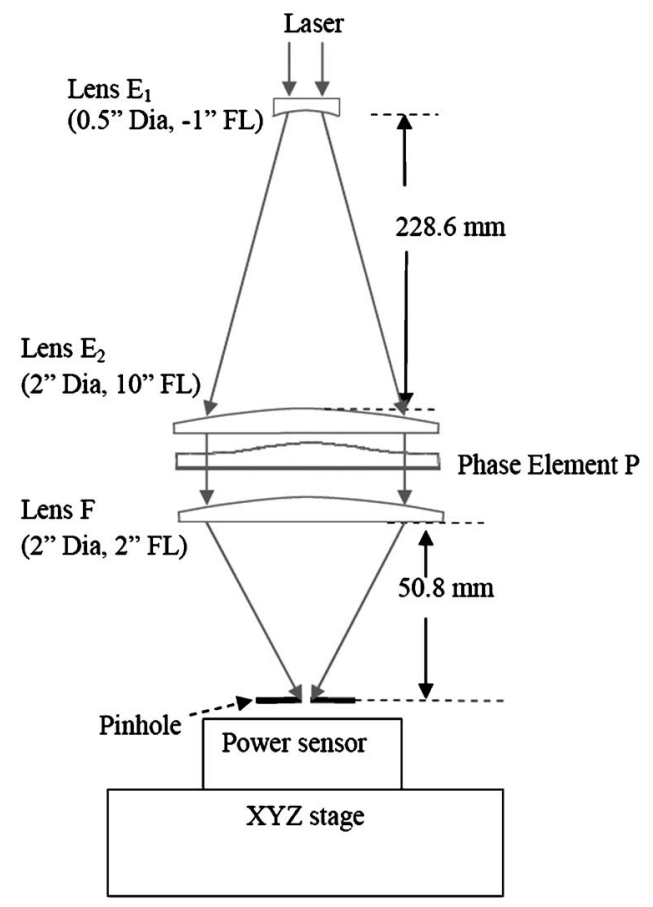

Fig. 13 Experimental setup for pitchfork beam profile measurement using diffractive optics (lenses $E_{1}$ and $E_{2}$ and phase element $P$ ) and a $50.8 \mathrm{~mm}$ focal length aspheric lens $\mathrm{F}$.

an aspheric lens of $50.8 \mathrm{~mm}$ focal length in the experimental setup shown in Fig. 13. The measured irradiance profiles have the pitchfork shape at different positions as shown in Fig. 14. It shows that the measured beam profiles are slightly asymmetric and the diameters are larger than the designed beam diameters. The asymmetric profile could be due to the imprecision in manufacturing the optics surfaces by diamond turning and the poor beam quality $\left(M^{2}=1.3\right)$ of the laser system. To improve the symmetry of the pitchfork beam, better optics fabrication techniques, such as photolithography and electron-beam lithography, can be used and laser systems with better beam quality can be investigated. Because the purpose of this paper is to provide a costeffective method for pitchfork beam shaping, these improvements were not pursued. The larger beam diameter could be due to aberration by the focusing lens, error in manufacturing the optics surfaces, beam quality of the laser system, and the effect of polarization on focusing the beam. The polarization effect requires a rigorous diffraction analysis based on the electric-field vector (complete Maxwell equations) instead of the scalar diffraction analysis used in the present case. The beam quality involves a detailed analysis of the electromagnetic field of the laser produced by the laser system, which is not investigated in this study.

The irradiance profile produced by the diffractive optical elements is compared to the one produced by refractive optics $^{10}$ in Fig. 15. Because the effect of diffraction is controlled by the diffractive optical elements, the amounts of laser energy in the diffraction side lobes are reduced significantly, as shown in Fig. 15. This reduction of energy in the side lobes is extremely important in laser microvia drill-

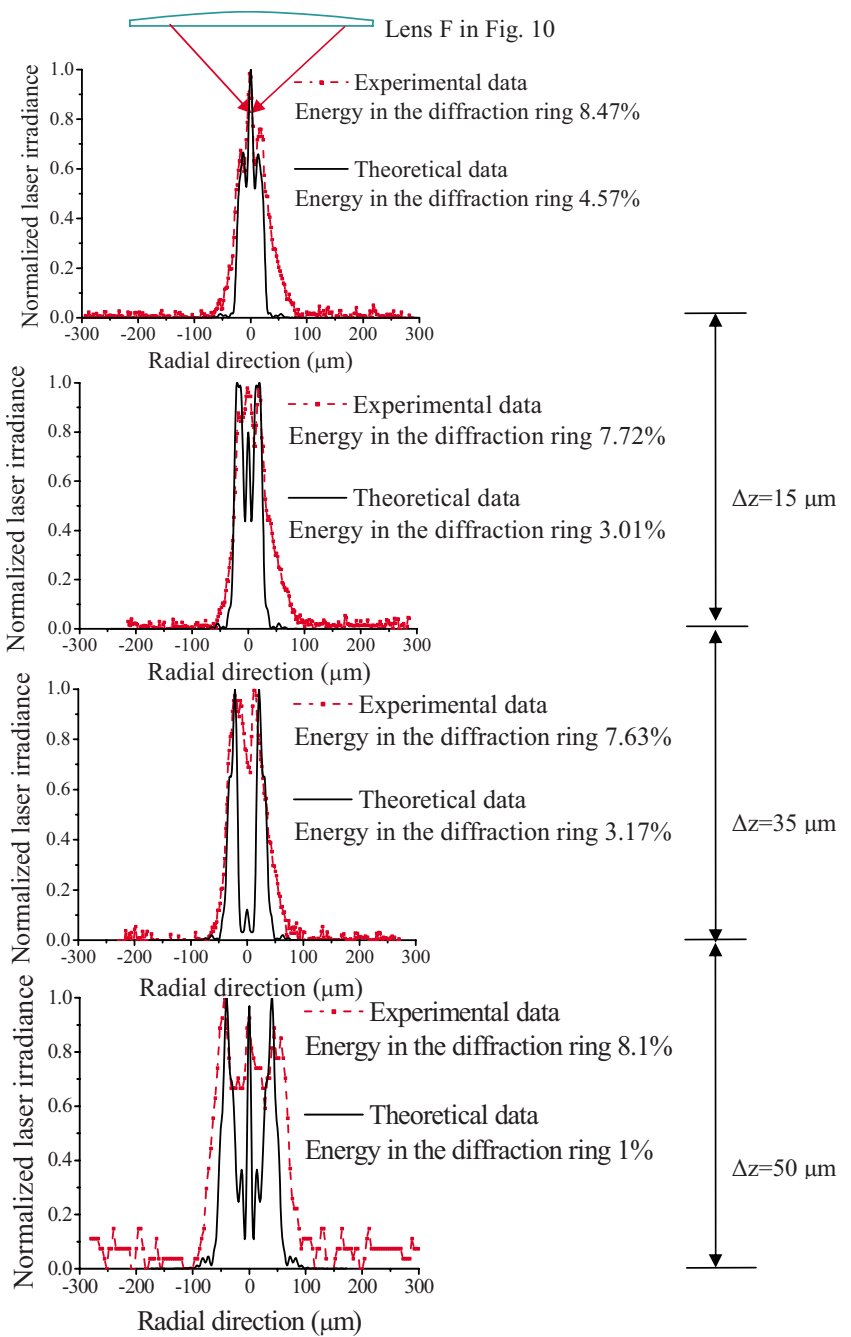

Fig. 14 Comparison between the optical model results and experimental data for the beam profiles with the beam shaping system.

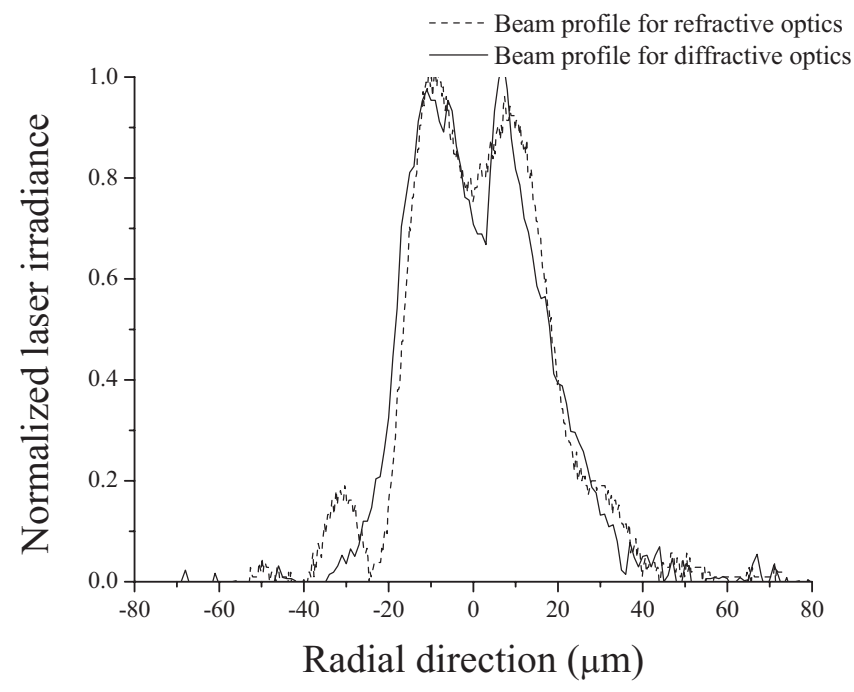

Fig. 15 Optimized optics reduced the laser energy contained in the diffraction ring 


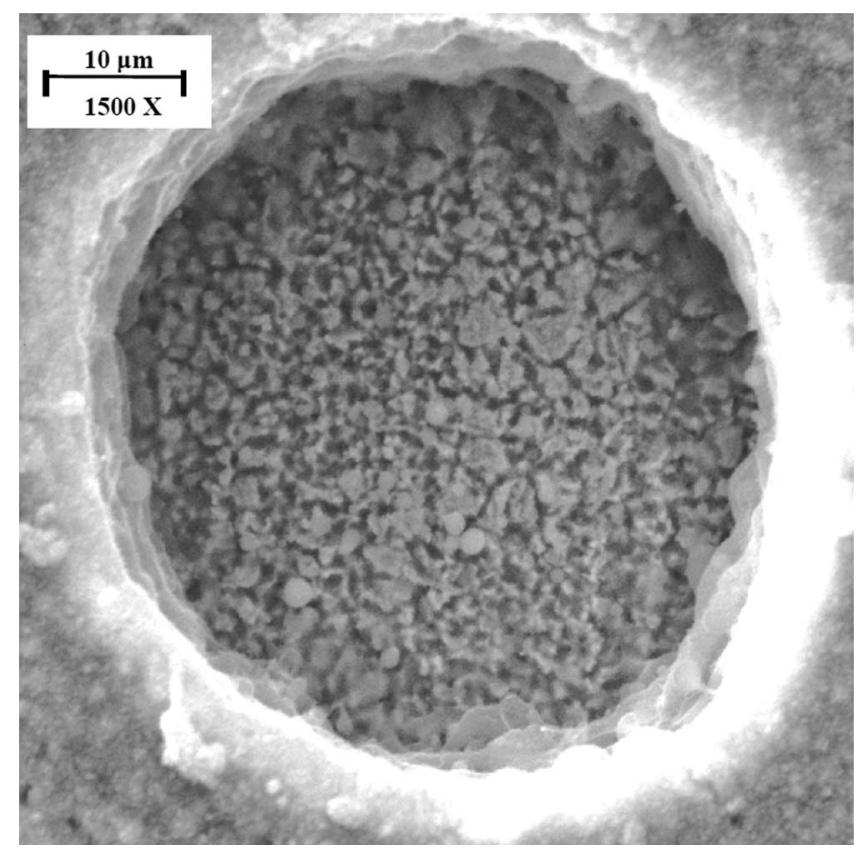

Fig. 16 Scanning electron microscopic top view of a microvia drilled with the following parameters: pulse energy $=0.276 \mathrm{~mJ}$, repetition rate $=100 \mathrm{kHz}$, five pulse trains, nine pulses in each pulse train, pulse length $=430 \mathrm{~ns}$, and time delay for each train is $1 \mathrm{~ms}$.

ing because it enables drilling high-quality microvias with a less heat-affected zone and high throughput rate.

\section{Microvia Drilling Experiment}

Small-diameter microvias were drilled in a polymeric substrate with a pitchfork beam of the above-mentioned $\mathrm{CO}_{2}$ laser. The substrate was made of two silica-filled polymer composite sheets of thickness $40 \mu \mathrm{m}$ each containing a copper film between them. The top view of a microvia based on scanning electron microscopy is presented in Fig. 16, where the copper film can be seen at the bottom. The granular microstructures in the copper film do not indicate any damage to the film by the laser beam during the drilling process. Such microstructures are inherent in the embedded copper film because the electroless process, which is used to deposit the film, produces granular grains instead of rolled copperlike smooth microstructures.

The microvia drilling parameters are pulse energy $=0.276 \mathrm{~mJ}$, repetition rate $=100 \mathrm{kHz}$, five pulse trains, nine pulses in each pulse train, pulse length $=430 \mathrm{~ns}$, time delay for each train is $1 \mathrm{~ms}$. The corresponding outputs are drilling time $=5.45 \mathrm{~ms}$, throughput rate $=183$ holes $/ \mathrm{s}$ with top and bottom diameters of 53 and $47 \mu \mathrm{m}$, respectively and tapering angle $=4.3 \mathrm{deg}$. Here, the tapering angle is defined as $\tan \theta=\left[\left(D_{t}-D_{b}\right) / 2\right] / D^{*}$, where $D_{t}$ and $D_{b}$ are the diameters at the top and bottom of the via respectively, and $D^{*}$ is the depth of the via. The tapering angle is reduced compared to the previous ${ }^{10}$ experimental data $(5-10 \mathrm{deg})$. Low tapering angle indicates that the residue in the corner at the bottom of the microvias can be removed efficiently using pitchfork beam profiles.

\section{Conclusions}

Diffractive optical elements are designed by an iterative method using adaptive additive algorithm to obtain a predetermined pitchfork beam from a given Gaussian profile. The entire optical system consists of a beam expander, a phase element, and a focusing lens to effect this transformation. The pitchfork irradiance profile is formed on the focal plane of the focusing lens. The beam-shaping optics enables reducing the amount of energy in the diffraction side lobes and increasing the amount of energy in the central lobe of the beam. The irradiance profile and diameter of the calculated and desired pitchfork beams match better if the focused beam diameter is much larger than the Airy disk diameter on the focal plane of the focusing lens. The depth of focus can also be tailored by designing appropriate beam-shaping optics. The energy in the diffraction rings and the depth of focus are two important properties of laser beams in microvia drilling for high-density microelectronics applications.

\section{Acknowledgment}

This work was supported by AppliCote Associates, LLC.

\section{References}

1. F. M. Dickey and S. C. Holswade, Laser Beam Shaping: Theory and Technique, Marcel Dekker, New York (2000).

2. T. Kajava, A. Hakola, H. Elfstrom, J. Simonen, P. Paakkonen and J. Turunen, "Flat-top profile of an excimer-laser beam generated using beam-splitter gratings," Opt. Commun. 268, 289-293 (2006).

3. F. D. Dickey, S. C. Holswade, and D. L. Shearly, Laser Beam Shaping Applications, CRC Press, New York (2005).

4. J. A. Hoffnagle and C. M. Jefferson, "Beam shaping with a planoaspheric lens pair," Opt. Eng. 42, 3090-3099 (2003).

5. M. Arif, M. M. Hossain, A. S. Awwal, and M. N. Islam, "Twoelement refracting system for annular Gaussian-to-Bessel beam transformation," Appl. Opt. 37, 4206-4209 (1998).

6. D. Zeng, W. P. Latham, and A. Kar, "Shaping of annular laser intensity profiles and their thermal effects for optical trepanning," Opt. Eng. 45(1), 014301 (2006).

7. J. Liu, M. Thomson, A. J. Waddie, and M. R. Taghizadeh, "Design of diffractive optical elements for high-power laser applications," Opt. Eng. 43, 2541-2548 (2004).

8. Y. A. Abdelaziez, P. P. Banerjee, and D. R. Evans, "Beam shaping by use of hybrid acousto-optics with feedback," Appl. Opt. 44, 34733481 (2005).

9. A. Shevchenko, A. Hakola, S. C. Buchter, M. Kaivola, and N. V. Tabiryan, "Laser beam shaping using self-focusing in a nematic liquid crystal," Mol. Cryst. Liq. Cryst. 454, 217-224 (2006).

10. C. Zhang, N. R. Quick, and A. Kar, "Pitchfork Beam Shaping for Laser microvias drilling," J. Phys. D 41, 125105 (2008).

11. J. R. Fienup, "Iterative method applied to image reconstruction and to computer-generated holograms," Opt. Eng. 19, 197-305 (1980).

12. R. W. Gerchberg and W. O. Saxton, "A practical algorithm for the determination of phase from image and diffraction plane pictures," Optik (Jena) 35, 237-246 (1972).

13. Z. Zalevsky, D. Mendlovic, and R. G. Dorsch, "Gerchberg-Saxton algorithm applied in the fractional Fourier or theFresnel domain," Opt. Lett. 21, 842-844 (1996).

14. M. A. Seldowitz, J. P. Allebach, and D. W. Sweeney, "Synthesis of digital holograms by direct binary search," Appl. Opt. 28, 2788-2798 (1987).

15. S. Kirkpatrick, C. D. Gelatt, and M. P. Vecchi, "Optimization by simulated annealing," Science 220, 671-680 (1983).

16. J. Turunen, A. Vasara, and J. Westerholm, "Kinoform phase relief synthesis: a stochastic method," Opt. Eng. 28, 1162-1167 (1987).

17. S. N. Dixit, J. K. Lawson, K. R. Manes, H. T. Powell, and K. A. Nugent, "Kinoform phase plates for focal plane irradiance profile control," Opt. Lett. 19, 417-419 (1994).

18. S. N. Dixit, M. D. Feit, M. D. Perry, and H. T. Powell, "Designing fully continuous phase screens for tailoring focal-plane irradiance profiles," Opt. Lett. 21, 1715 (1996).

19. Y. Lin, T. J. Kessler, and G. N. Lawrence, "Design of continuous surface-relief phase plates by surface-based simulated annealing to achieve control of focal-plane irradiance," Opt. Lett. 21, 1703 (1996).

20. J. S. Liu and M. R. Taghizadeh, "Iterative algorithm for the design of 
diffractive phase elements for laser beam shaping," Opt. Lett. 27, 1463 (2002).

21. X. H. Guang, M. R. Wang, and C. Yu, "High-efficiency flat-top beam shaper fabricated by a non-lithographic technique," Opt. Eng. 38, 208-213 (1999).

22. V. A. Soifer, Methods for Computer Design of Diffractive Optical Elements, Wiley, Hoboken, NJ, p. 60 (2002).

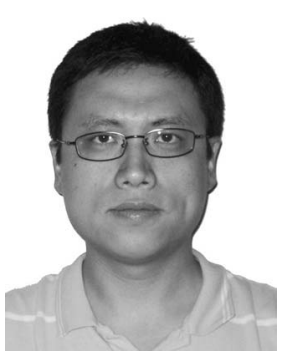

Chong Zhang received his $\mathrm{PhD}$ in mechanical engineering from the University of Central Florida in 2008. He is currently a laser application engineer in Resonetics, Inc. His research interests are in the areas of laser-matter interactions, laser materials processing, laser micromachining, and laser beam shaping.

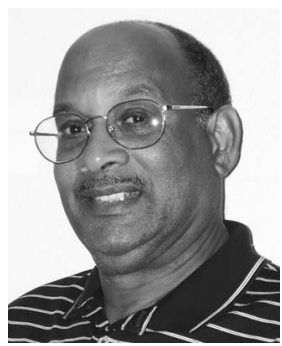

Nathaniel R. Quick is the founder, president, and chief technical officer of AppliCote Associate, LLC, a technology development company. He established the company to develop laser-based technologies that enable the next generation of semiconductor devices. He has held technology positions from materials scientist to vice president of R\&D for fortune 100 companies and has managed multimillion dollar new process and product development projects and campaigns. Dr. Quick has a PhD form Cornell University in materials science and engineering and is the president-elect, past secretary, board member, and fellow of the Laser Institute of America. He is a fellow of the African Scientific Institute, a past guest researcher at NIST, and serves on the advisory board of the UCF Florida Photonics Center of Excellence and the UCF Industrial Advisory Committee. He currently holds 39 U.S. patents and has over 60 publications.

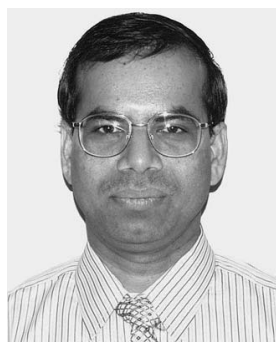

Aravinda Kar received his $\mathrm{PhD}$ in nuclear engineering from the University of Illinois at Urbana-Champaign in 1984. His research interest includes laser-matter interactions, laser science and technology, laser beam properties, laser beam shaping, optoelectronic devices (such as light-emitting diodes and sensors), semiconductor optics, laser materials processing, materials synthesis, and process modeling and simulation. $\mathrm{He}$ has coauthored a book entitled, Theory and Application of Laser Chemical Vapor Deposition (Plenum Press, New York, 1995). 